\title{
Cosmological Constraints from the Redshift Dependence of the Alcock-Paczynski Effect: Dynamical Dark Energy
}

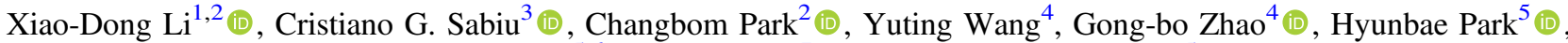 \\ Arman Shafieloo ${ }^{5,6}$ (D), Juhan $\mathrm{Kim}^{7}$ (D), and Sungwook E. Hong ${ }^{5}$ (iD \\ ${ }^{1}$ School of Physics and Astronomy, Sun Yat-Sen University, Guangzhou 510297, People's Republic of China \\ ${ }^{2}$ School of Physics, Korea Institute for Advanced Study, 85 Heogiro, Dongdaemun-gu, Seoul 02455, Republic of Korea \\ ${ }^{3}$ Department of Astronomy, Yonsei University, 50 Yonsei-ro, Seoul 03722, Republic of Korea; csabiu@ gmail.com \\ ${ }^{4}$ National Astronomy Observatories, Chinese Academy of Science, Beijing, 100012, People's Republic of China \\ ${ }^{5}$ Korea Astronomy and Space Science Institute, 776, Daedeokdae-ro, Yuseong-gu, Daejeon 34055, Republic of Korea \\ ${ }^{6}$ University of Science and Technology (UST), Yuseong-gu 217 Gajeong-ro, Daejeon 34113, Republic of Korea \\ ${ }^{7}$ Center for Advanced Computation, Korea Institute for Advanced Study, 85 Hoegi-ro, Dongdaemun-gu, Seoul 02455, Republic of Korea \\ Received 2017 November 5; revised 2018 February 12; accepted 2018 March 3; published 2018 March 28
}

\begin{abstract}
We perform an anisotropic clustering analysis of 1,133,326 galaxies from the Sloan Digital Sky Survey (SDSS-III) Baryon Oscillation Spectroscopic Survey Data Release 12 covering the redshift range $0.15<z<0.69$. The geometrical distortions of the galaxy positions, caused by incorrect assumptions in the cosmological model, are captured in the anisotropic two-point correlation function on scales of 6-40 $\mathrm{h}^{-1} \mathrm{Mpc}$. The redshift evolution of this anisotropic clustering is used to place constraints on the cosmological parameters. We improve the methodology of $\mathrm{Li}$ et al. to enable efficient exploration of high-dimensional cosmological parameter spaces, and apply it to the Chevallier-Polarski-Linder parameterization of dark energy, $w=w_{0}+w_{a} z /(1+z)$. In combination with data on the cosmic microwave background, baryon acoustic oscillations, Type Ia supernovae, and $H_{0}$ from Cepheids, we obtain $\Omega_{m}=0.301 \pm 0.008, w_{0}=-1.042 \pm 0.067$, and $w_{a}=-0.07 \pm 0.29$ (68.3\% CL). Adding our new Alcock-Paczynski measurements to the aforementioned results reduces the error bars by $\sim 30 \%-40 \%$ and improves the dark-energy figure of merit by a factor of $\sim 2$. We check the robustness of the results using realistic mock galaxy catalogs.
\end{abstract}

Key words: cosmological parameters - dark energy - large-scale structure of universe

\section{Introduction}

The origin of the late-time accelerating expansion of the universe is one of the most salient questions in contemporary cosmology. Theoretical explanations for this phenomenon are numerous and range from a non-zero vacuum energy, an evolving scalar field remnant from the Big Bang, to modifications of Einstein's General Relativity (Li et al. 2011; Yoo \& Watanabe 2012; Joyce et al. 2015). Considering the wealth of theoretical explanations, it is crucial to obtain precise and unbiased measurements of the expansion history of the universe, which will allow us to differentiate between competing models.

In recent years the Alcock-Paczynski (AP) test (Alcock \& Paczynski 1979), applied to samples of galaxy redshifts (Outram et al. 2004; Blake et al. 2011; Alam et al. 2017), has allowed tight constraints to be placed on the backgroundaveraged distance scales, $D_{A}(z)$ and $H^{-1}(z)$. Assuming an incorrect cosmological model for the coordinate transformation between redshift space and comoving space produces residual geometric distortions in the resultant galaxy distribution as well as a change in volume elements (Park \& Kim 2010); see Figure 1 as an illustrative example. These distortions are induced by the fact that distances measured along and perpendicular to the line of sight (LoS) depend on the given cosmological parameters. Therefore, measuring the ratio of galaxy clustering in the radial and transverse directions provides a probe of this AP effect, which is sensitive to the product $D_{A}(z) H(z)$.

The main caveat in applying the AP test is that the radial distances of galaxies are inferred from observed redshifts. Thus
AP tests are inevitably affected by the peculiar motions of galaxies, which leads to apparent anisotropy in the clustering signal, even if the adopted cosmology is correct. This effect, known as redshift-space distortions (RSD), is notoriously difficult to model accurately in the statistics of galaxy clustering (Ballinger et al. 1996).

The symmetry properties of galaxy pairs (Marinoni \& Buzzi 2010; Bueno Belloso et al. 2012) could also be used to probe the AP effect; however, since the peculiar velocity distorts the redshifts and changes the apparent tilt angles of galaxy pairs, this method is also seriously limited by RSD (Jennings et al. 2011).

In an effort to minimize RSD contamination, the shape of void regions (Ryden 1995; Lavaux \& Wandelt 2012; Hamaus et al. 2016) has been proposed as an AP probe. This approach has the advantage that the void regions are easier to model than dense regions, but has limitations in that it utilizes only lowdensity regions of the large scale structure (LSS) and requires large samples to attain statistical significances and achieve competitive constraints (Mao et al. 2017).

Previously, we proposed to use the redshift dependence of the AP distortion ( $\mathrm{Li}$ et al. 2014) as a way of mitigating the RSD effect. The clustering anisotropies produced by RSD, although large, are close to uniform in magnitude over a wide range in redshift. However, if cosmological parameters are incorrectly chosen and the AP effect exists, the anisotropy in the clustering signal has a clear redshift dependence (as an illustration, Figure 1 shows how the shape distortion varies with distance when incorrect cosmologies are used to infer distance from redshift). In Li et al. (2015), we developed an AP 


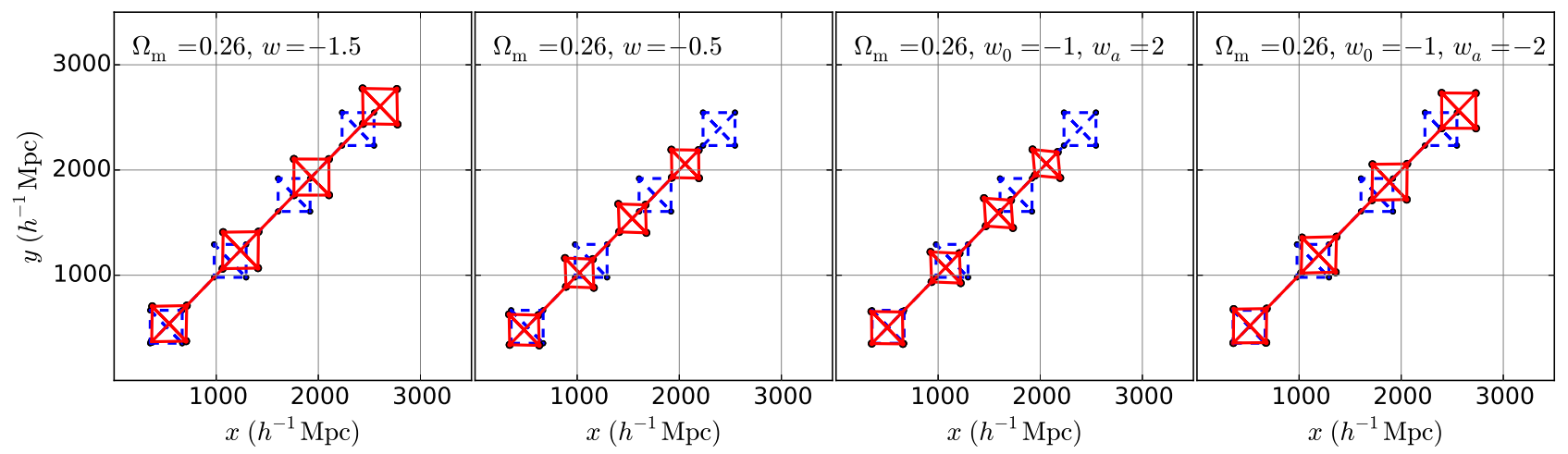

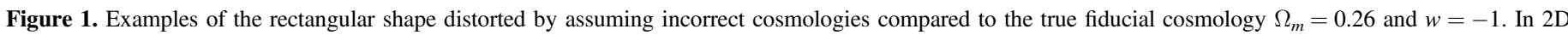

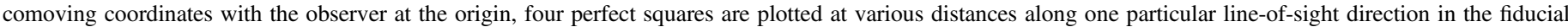

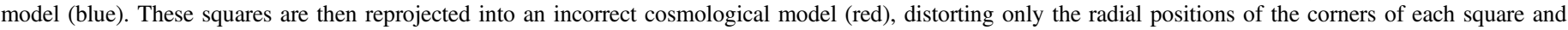
resulting in a distorted quadrilateral shape.

methodology that utilizes the redshift dependence of the galaxy two-point correlation function (2PCF), measured as a function of angle between the galaxy pair and the LoS.

In an earlier work (Li et al. 2016, hereafter L16) we applied this AP method to galaxies from the Sloan Digital Sky Survey (SDSS-III), data release (DR) 12. Combining the method with measurements of the cosmic microwave background (CMB), Type Ia supernovae ( $\mathrm{SNe}$ Ia), baryon acoustic oscillations (BAO), and $H_{0}$, we obtained very tight constraints of $\Omega_{m}=0.301 \pm 0.006$ and $w=-1.054 \pm 0.025$. In reducing the RSD effect, we were able to use galaxy clustering on scales down to $6 h^{-1} \mathrm{Mpc}$, which is a major advance in extracting cosmological information on small scales where galaxy clustering is strong and many independent structures exist.

In this paper, we continue to develop our previous methodology and proceed to set the constraints on dynamical dark energy. We will use the same observational data as in L16, but we present an improved methodology compared to L16, allowing for faster likelihood estimation and thus the exploration of larger, higher dimensional parameter spaces. The methodology we will present here can be applied to any model of dynamical dark energy, or indeed any appropriately chosen parametric or non-parametric decomposition of the cosmic expansion history. However, as a first step, in this paper we will focus on the widely used Chevallier-Polarski-Linder (CPL) parameterization (Chevallier \& Polarski 2001; Linder 2003),

$$
w(z)=w_{0}+w_{a}(1-a)=w_{0}+w_{a} \frac{z}{1+z} .
$$

This parameterization characterizes the equation of state (EoS) of dark energy by two free parameters: $w_{0}$ determines the present-day value, while $w_{a}$ characterizes the first-order derivative of $w$ with respect to $a$. The possible redshift evolution of the EoS of dark energy is not considered in the analysis of L16.

The CPL parameterization has many obvious advantages, for instance, a manageable parameter space, the bounded behavior at high redshift, and the ability to accurately reconstruct many theories of dark energy (Linder 2003). The constraining power is usually quantified by the Dark Energy Task Force (Albrecht et al. 2006) figure of merit, defined as the reciprocal of the area of the error ellipse enclosing the $95 \%$ confidence limit (CL) in the $w_{0}-w_{a}$ plane.
The rest of this paper is arranged as follows: in Section 2 we describe the data that are used and outline the methodology for obtaining cosmological constraints. In Section 3 we present the main findings of this study, and finally we conclude in Section 4.

\section{Methodology}

The methodology follows closely that of our previous work (Li et al. 2016), where we used the redshift dependence of the anisotropic clustering of galaxies to test cosmological models. When transforming galaxy positions in \{R.A., decl., redshift $\}$ to comoving cartesian coordinates we must assume a cosmological model. Any difference between our assumed model and the true model will induce geometrical distortions on the resultant galaxy distribution (AP effect). This can be more easily visualized in Figure 1, where we illustrate the AP effect in four incorrect cosmologies.

In this toy model, the boxes in the fiducial cosmology (blue) are reprojected into different cosmologies (red) with various choices of $\Omega_{m}, w_{0}$, and $w_{a}$. As we can see from the figure, varying the cosmology alters the position, size, and shape of the boxes in a redshift-dependent fashion. Thus, we may expect that the shape of the clustering statistics will also be affected in a similar way.

In Li et al. (2016) we considered only non-evolving models of dark energy. However, since a redshift dependence of the shape distortion is observed when adopting incorrect values of $w_{0}$ and $w_{a}$, we expect in Figure 1 that these two parameters will be sensitive to our method.

\subsection{Data}

We use the spectroscopic galaxy sample of SDSS-III BOSS (Baryon Oscillation Spectroscopic Survey), which has two primary catalogs: the LOWZ sample, designed as an extension of the SDSS-I/II luminous red galaxy sample to $z \approx 0.4$ and fainter luminosities, and the CMASS sample covering a higher range $(0.4 \lesssim z \lesssim 0.7)$ and made to be an approximately stellar masslimited sample of massive, luminous galaxies (Reid et al. 2016). In the clustering analysis we use 1,133,326 galaxies, split into six, non-overlapping redshift bins, $0.150<z_{1}<0.274<z_{2}<$ $0.351<z_{3}<0.430<z_{4}<0.511<z_{5}<0.572<z_{6}<0.693$. The edges are determined so that the numbers of galaxies are roughly the same in different redshift bins (for LOWZ and CMASS samples, respectively). 


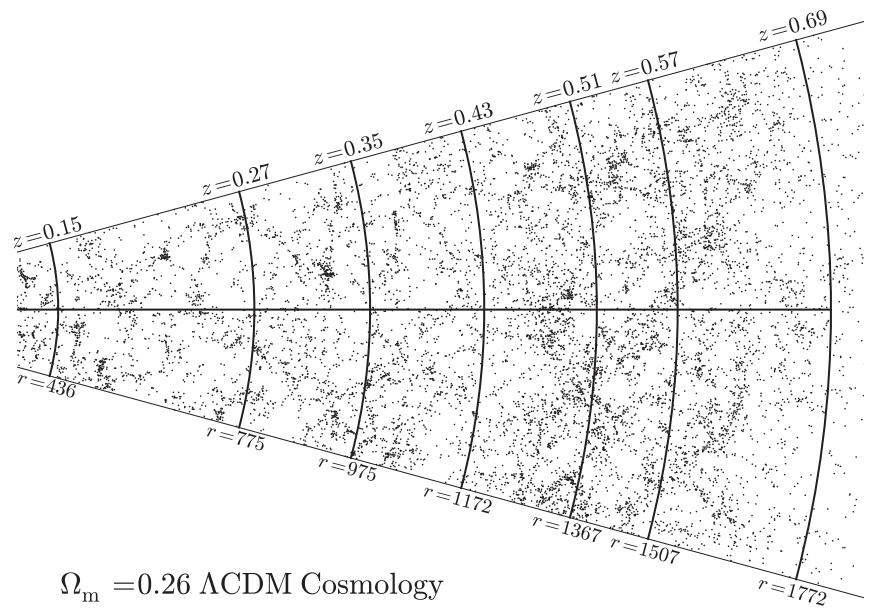

Figure 2. A patch of SDSS BOSS DR12 galaxies in the fan-shaped region of $140^{\circ}<$ R.A. $<170^{\circ}, 10^{\circ}<$ decl. $<13^{\circ}$, split into six non-overlapping redshift bins (marked by the arcs) in order to probe the redshift evolution of anisotropic clustering. We plot the galaxy positions computed using $\Omega_{m}=0.26$ $\Lambda \mathrm{CDM}$. Redshifts and comoving distances (in units of $h^{-1} \mathrm{Mpc}$ ) of the edges of redshift bins are listed.

Figure 2 shows a patch of 10,976 BOSS DR12 galaxies, whose positions are computed in the $\Omega_{m}=0.26 \Lambda \mathrm{CDM}$ cosmology. By investigating how the anisotropy of galaxy distribution evolves in the six redshift bins, we are able to distinguish particular cosmological models.

\subsection{Quantifying the Redshift Dependence of the AP Distortion}

Following our previous methodology, the information on anisotropic clustering is computed ${ }^{8}$ as

$$
\xi_{\Delta s}(\mu) \equiv \int_{s_{\min }}^{s_{\max }} \xi(s, \mu) d s,
$$

with $s_{\min }=6 h^{-1} \mathrm{Mpc}$ and $s_{\max }=40 h^{-1} \mathrm{Mpc}$. We then normalize these clustering shells as

$$
\hat{\xi}_{\Delta s}(\mu) \equiv \frac{\xi_{\Delta s}(\mu)}{\int_{0}^{\mu_{\max }} \xi_{\Delta s}(\mu) d \mu}
$$

to nullify the amplitude information of the clustering signal, which is not associated with the AP test and is mostly sensitive to evolution of the galaxy bias. The "correct" cosmological model is selected by minimizing the amount of redshift evolution of $\hat{\xi}_{\Delta s}$, via a $\chi^{2}$ function,

$$
\chi^{2} \equiv \sum_{i=2}^{6} \sum_{j_{1}=1}^{n_{\mu}} \sum_{j_{2}=1}^{n_{\mu}} \boldsymbol{p}\left(z_{i}, \mu_{j_{1}}\right)\left(\mathbf{C o v}_{i}^{-1}\right)_{j_{1}, j_{2}} \boldsymbol{p}\left(z_{i}, \mu_{j_{2}}\right)
$$

where $\boldsymbol{p}\left(z_{i}, \mu_{j}\right)$ is the redshift evolution of clustering with respect to the lowest redshift bin, while subtracting systematic effects:

$$
\begin{aligned}
\boldsymbol{p}\left(z_{i}, \mu_{j}\right) \equiv & {\left[\hat{\xi}_{\Delta s}\left(z_{i}, \mu_{j}\right)-\hat{\xi}_{\Delta s}\left(z_{1}, \mu_{j}\right)\right] } \\
& -\left[\hat{\xi}_{\Delta s}\left(z_{i}, \mu_{j}\right)-\hat{\xi}_{\Delta s}\left(z_{1}, \mu_{j}\right)\right]_{\mathrm{sys}}
\end{aligned}
$$

\footnotetext{
8 These correlations were computed using the public code KSTAT https:// bitbucket.org/csabiu/kstat.
}

We use $n_{\mu}=20,21, \ldots, 25$ bins for $0<\mu<\mu_{\text {max }}$. To reduce the fiber collision and the Finger of God (FoG) effect (Jackson 1972) near the LoS we take a cut $\mu_{\max }=0.97$.

The systematic effects are estimated using mock catalogs drawn from Horizon Run 4 (HR4; Kim et al. 2015), an $N$-body simulation with a box size of $L=3150 h^{-1} \mathrm{Mpc}$, the number of particles $6300^{3}$, initial redshift of $z_{i}=100$, and the WMAP5 (Komatsu et al. 2011) cosmological parameters $\left(\Omega_{b}, \Omega_{m}, \Omega_{\Lambda}, h\right.$, $\left.\sigma_{8}, n_{s}\right)=(0.044,0.26,0.74,0.72,0.79,0.96)$. Mock galaxy samples are produced using a modified version of the one-toone correspondence scheme (Hong et al. 2016).

Since the mock catalogs were analyzed using the cosmology with which they were run, they have no geometrical distortions associated with the AP effect, allowing us to focus solely on modeling the RSD effect.

The covariance matrix, Cov, is computed from a set of 2000 MultiDark PATCHY mock catalogs (Kitaura et al. 2016). The statistical bias and scattering in the likelihood function (due to the finite number of mocks in the estimation of covariance) are adequately corrected (Hartlap et al. 2007; Percival et al. 2014).

The MultiDark PATCHY mocks are produced using approximate gravity solvers and analytical-statistical biasing models. They were calibrated to the BigMultiDark $N$-body simulation (Klypin et al. 2016), which uses $3840^{3}$ particles in a volume of $\left(2.5 h^{-1} \mathrm{Gpc}\right)^{3}$, assuming a $\Lambda$ CDM cosmology with $\left(\Omega_{b}, \Omega_{m}, h, \sigma_{8}, n_{s}\right)=(0.048206,0.307115,0.6777,0.8288$, $0.9611)$. The mock surveys can well reproduce the number density, selection function, survey geometry, and $2 \mathrm{PCF}$ measurement of the BOSS DR12 catalogs. They have been adopted for statistical analysis of BOSS data in a series of works (see Alam et al. 2017, and references therein).

As an illustration, Figure 3 shows how we use the above procedure to distinguish different cosmologies. Here we plot the value of $\hat{\xi}_{\Delta s}(\mu)$ (upper panels) as well as its redshift evolution (lower panels), measured from the BOSS DR12 galaxies in six redshift bins. Two cosmologies are adopted, one with $\Omega_{m}=0.26$ and the other with a strongly disfavored value of $w_{a}=-2$.

The shape of $\hat{\xi}_{\Delta s}(\mu)$ is very different from a flat curve, due to the apparent anisotropy produced by the peculiar motion of galaxies. In the $w_{a}=-2$ cosmology, the shapes of $\hat{\xi}_{\Delta s}(\mu)$ are different from the measurements in the $\Omega_{m}=0.26$ cosmology, and the amount of difference evolves systematically with redshift. We observe a large redshift evolution of $\hat{\xi}_{\Delta s}(\mu)$, indicating that it is not likely to be the underlying true cosmology of our universe.

The green curves denote the 2PCFs measured from the HR4 mock catalogs (we plot the correct measurement in the simulation cosmology, i.e., the $\Omega_{m}=0.26 \Lambda \mathrm{CDM}$ ) and have not been corrected for systematics. So their amplitude simply represents the magnitude of the systematic effects.

The simulation results can match the general shape of the results from observational data, indicating that the FoG (Jackson 1972) and Kaiser (1987) effects are both well reproduced. Since there is no AP effect in the simulation measurements, all detected redshift evolution should be due to effects other than the cosmological effect; so they are adopted as an estimation of the systematic effects of the method. The amount of systematics reaches $4 \%-6 \%$ in the sixth redshift bin, and is much smaller $(\lesssim 2 \%)$ in the other bins.

In L16, the likelihood contour of $\Omega_{m}-w$ was constructed by measuring the 2PCF 3195 times, using 3D positions of BOSS 
Cosmology to interpret SDSS data changed to $\Omega_{\mathrm{m}}=0.26, w_{0}=-1, w_{a}=-2$

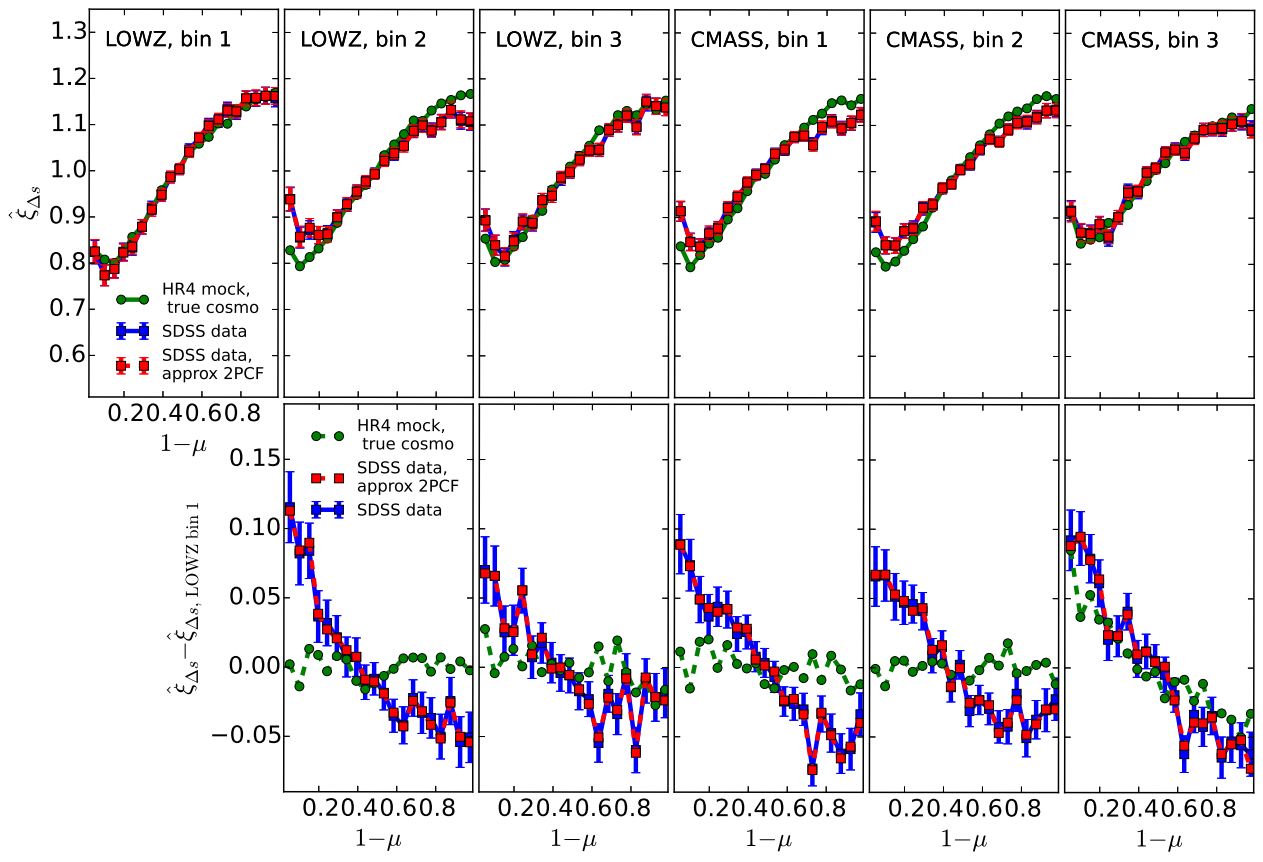

Cosmology : $\Omega_{\mathrm{m}}=0.26 \Lambda \mathrm{CDM}$

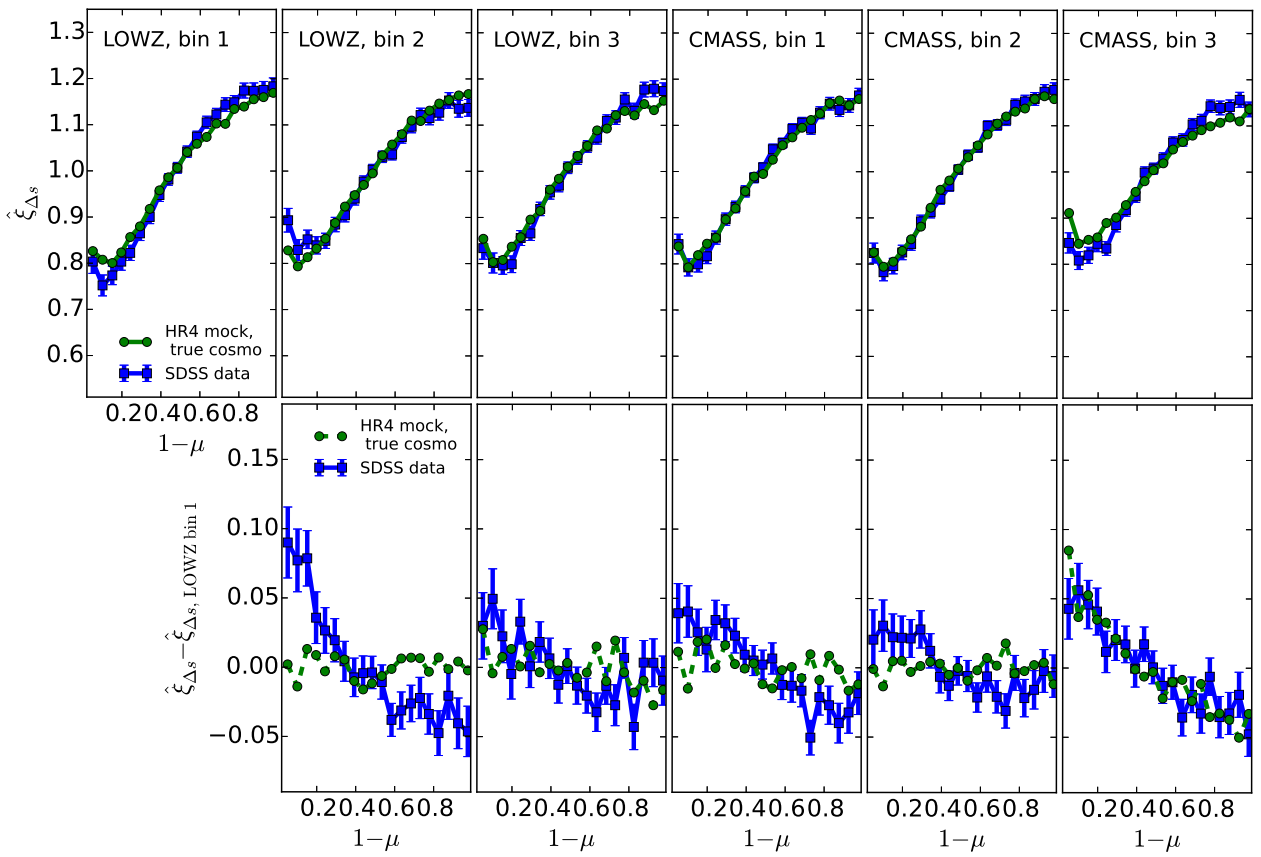

Figure 3. $\hat{\xi}_{\Delta s}(\mu)$ measured from the SDSS BOSS DR12 galaxies in six redshift bins (three in LOWZ and three in CMASS), assuming the $\Omega_{m}=0.26 \Lambda$ CDM cosmology and a more dark-energy-dominated cosmology with $w_{a}=-2$. Measurements, without systematic correction, are plotted for each of the six redshift bins and their redshift evolution with respect to the first bin of LOWZ. In the $w_{a}=-2$ cosmology, the shapes of $\hat{\xi}_{\Delta s}(\mu)$ are different from the results of the $\Omega_{m}=0.26$ cosmology, and the difference changes with redshift; a large redshift evolution of $\hat{\xi}_{\Delta s}(\mu)$ is detected in this cosmology, indicating that it is not likely to be the underlying true cosmology of our universe. The measurements in the HR4 mock catalogs (always in the $\Omega_{m}=0.26 \Lambda \mathrm{CDM}$ cosmology; plotted in green) match the general shape of curves measured from observational data, indicating that the simulation reproduces the FoG and Kaiser effects. For the $w_{a}=-2$ cosmology, we also plot the approximate $2 \mathrm{PCFs}$ (red dashed lines) inferred using the technique described in Appendix A. The error induced in the approximation procedure is very small.

galaxies computed in $71 \times 45$ sets of cosmological parameters. This procedure took $\sim 1$ month using 500 cores of the Korea Institute for Advanced Study Baekdu cluster. It would be computationally intractable to attempt a full Markov chain Monte Carlo (MCMC) calculation of all relevant cosmological parameters using this approach. Thus we adopt an "approximate 

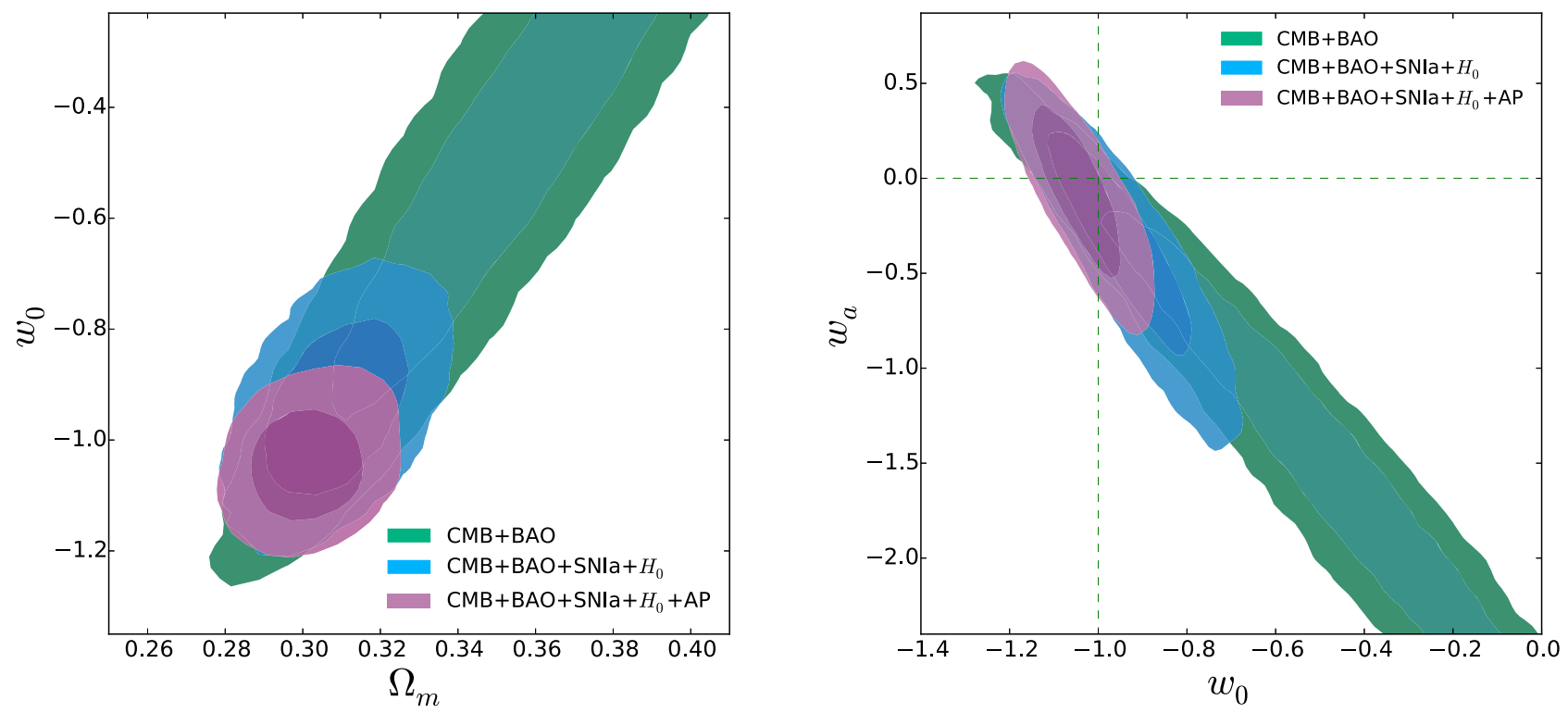

Figure 4. Cosmological parameter constraints on the CPL dark energy parameterization $w=w_{0}+w_{a} z /(1+z)$. The 68.3\% and 95.4\% CL likelihood contours are plotted in the $\Omega_{m}-w_{0}$ plane (left panel) and the $w_{0}-w_{a}$ plane (right panel). Results from CMB $+\mathrm{BAO}$ (cyan filled), $\mathrm{CMB}+\mathrm{BAO}+\mathrm{SNe}$ Ia $+H_{0}$ (blue filled), and $\mathrm{CMB}+\mathrm{BAO}+\mathrm{SNe} \mathrm{Ia}+H_{0}+\mathrm{AP}$ (magenta filled) are shown. Adding our AP method to the $\mathrm{CMB}+\mathrm{BAO}+\mathrm{SNe}$ Ia $+H_{0}$ combination reduces the contour area by as much as $50 \%$.

2PCF" by transforming our measurements from one cosmology to another. A detailed explanation of this procedure is given in Appendix A.

\section{Cosmological Constraints}

The Planck team has released the COSMOMC (Lewis \& Bridle 2002) outputs of four MCMC "chains" in the CPL model, using a combination of four data sets: the full-mission Planck observations of $\mathrm{CMB}$ temperature and polarization anisotropies (Ade et al. 2016); the BAO distance priors measured from SDSS DR11 (Anderson et al. 2014), 6dFGS (Beutler et al. 2011), and SDSS MGS (Ross et al. 2015); the "JLA" sample of SNe Ia (Betoule et al. 2014); and the Hubble Space Telescope measurement of $H_{0}=70.6 \pm 3.3 \mathrm{~km} \mathrm{~s}^{-1} \mathrm{Mpc}^{-1}$ (Riess et al. 2011; Efstathiou 2014). These MCMC chains contain the CMB $+\mathrm{BAO}+\mathrm{SNe} \mathrm{Ia}+H_{0}$ likelihood computed for $\sim 37,000$ sets of cosmological parameters. After adding the log-likelihoods of the Planck team sample to ours, while also multiplying the sample weights by our likelihoods, we derive the $\mathrm{CMB}+\mathrm{BAO}+\mathrm{SNe}$ Ia $+H_{0}+\mathrm{AP}$ constraints on CPL parameters.

\subsection{Results}

Figure 4 shows the $68.3 \%$ and $95.4 \%$ CL likelihood contours in the $\Omega_{m}-w_{0}$ and $w_{0}-w_{a}$ planes, derived from the CMB + $\mathrm{BAO}, \mathrm{CMB}+\mathrm{BAO}+\mathrm{SNe} \mathrm{Ia}+H_{0}$, and $\mathrm{CMB}+\mathrm{BAO}+\mathrm{SNe}$ $\mathrm{Ia}+H_{0}+\mathrm{AP}$. The overlapping of the various contours suggests that they are consistent with each other.

The current $\mathrm{CMB}+\mathrm{BAO}$ data sets are not statistically powerful enough to effectively constrain the $w_{0}-w_{a}$ parameter space. Combining the four external techniques, i.e., $\mathrm{CMB}+$ $\mathrm{BAO}+\mathrm{SNe}$ Ia $+H_{0}$, leads to effective constraints on all parameters. The statistical mean values and $68.3 \%$ uncertainties of these parameters are

$$
\begin{gathered}
\Omega_{m}=0.309 \pm 0.010, \\
w_{0}=-0.938 \pm 0.109,
\end{gathered}
$$

$$
w_{a}=-0.38 \pm 0.41 \text {. }
$$

Adding our AP method to this combination further tightens the constraints, leading to

$$
\begin{gathered}
\Omega_{m}=0.301 \pm 0.008, \\
w_{0}=-1.042 \pm 0.067, \\
w_{a}=-0.07 \pm 0.29 .
\end{gathered}
$$

The error bars are dramatically reduced by $30 \%-40 \%$, and the contour areas are reduced by $\sim 50 \%$, i.e., the dark-energy figure of merit is improved by $\sim 100 \%$. Notice that the AP constraints come from the BOSS DR12 data, which are already used in the $\mathrm{BAO}$ analysis. So the doubling of the figure of merit comes at no additional cost or alteration to data size, thus greatly improving the overall cost-benefit balance of the large cosmological redshift surveys.

Zhang et al. (2018) tested the correlation between the BAO and $\mathrm{AP}$ methods and find that the information extracted from each methods is statistically independent. The BAO method uses the BAO feature in the clustering of galaxies on scales of 100 $150 h^{-1} \mathrm{Mpc}$, created by the oscillation of the baryon-photon plasma in the early universe. Measuring the BAO feature in 1D or 2D then yields measurements of $D_{V}$ or $D_{A}$ and $H$ at some representative redshift. As a comparison, the AP method uses galaxy clustering on scales of $6-40 h^{-1} \mathrm{Mpc}$, which is much smaller than the BAO scale. The information explored from the two methods are fairly independent, so we can easily combine them without worrying about their correlation.

It can be also noted that, after adding the AP method, the central value of $w_{a}$ moved significantly toward zero. This implies that the accelerated expansion of the universe is driven by the cosmological constant or a dark energy component that has no evolution. Figure 5 shows the redshift evolution of $w(z)$ derived from the cosmological constraints. Adding the new AP results tightens the constraints and reduces the redshift evolution of $w$ (tilt of $w(z)$ ).

In Appendix B, these results are tested for robustness. We find that the results are unaffected by the $\operatorname{LoS} \mu$-cut, the range 


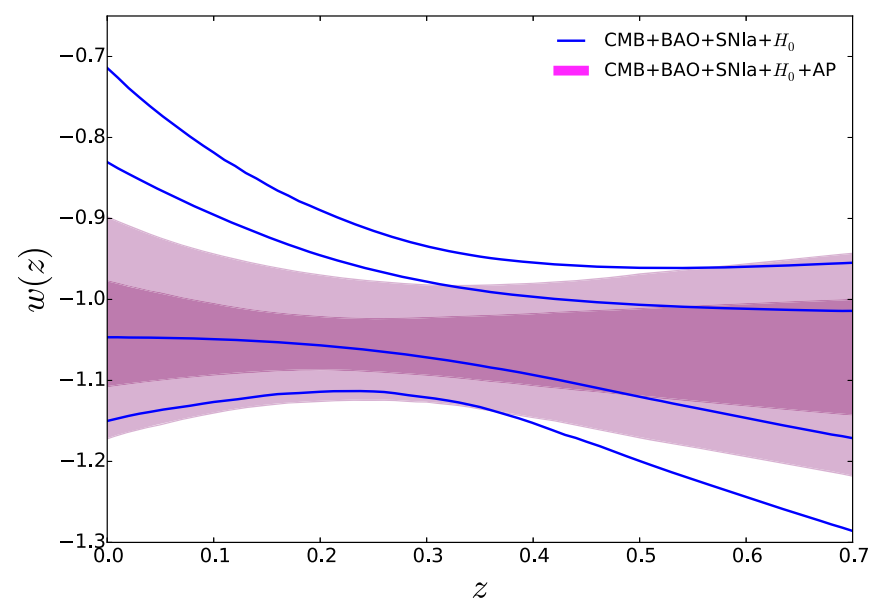

Figure 5. Derived redshift evolution of $w(z)$ : the $68.3 \%$ and $95.4 \%$ CL regions are plotted. Adding the AP method tightens the constraints and reduces the redshift evolution of $w$ (the tilt of $w(z)$ ).

of radial integration, the choice of fiducial cosmology in the mapping of $\xi(s, \mu)$, or the number of mocks.

\section{Conclusions}

In recent studies we have proposed to constrain cosmological parameters governing the expansion history of the universe via the redshift dependence of anisotropic galaxy clustering (Li et al. 2014, 2015, 2016). This approach enables a robust AP test on relatively small scales. In this paper we improved the methodology and obtained constraints on the CPL parameterization of dark energy. The derived cosmological constraints are fully consistent with a cosmological constant or a dark energy component with no evolution.

The AP method presented in this work has many advantages over the "traditional" methods using galaxy clustering. Since it works with the redshift evolution of the anisotropic clustering signal, it significantly reduces the effect of systematics. Our method mitigates many of the difficulties in accurately modeling the RSD, nonlinear clustering, and galaxy bias. This implementation of the AP test can use galaxy clustering statistics at smaller scales than other methods, thus including many more $k$-modes and maximizing the information gain.

In this analysis, we find that the systematic effects do not significantly affect the derived cosmological constraints. But it remains to be seen if this is true for future galaxy surveys. In particular, the systematic effects are estimated using simulations performed in one fiducial cosmology. The cosmological dependence of the systematics remains to be investigated in future works.

In this analysis, combining our method with the CMB + $\mathrm{SNe} \mathrm{Ia}+\mathrm{BAO}+\mathrm{H}_{0}$ data sets, the dark-energy figure of merit is improved by a factor of $\sim 2$. This indicates the great power of the method in constraining the cosmic expansion history and probing the properties of dark energy.

In Li et al. (2014, 2015) we tested our methodology and found that it is applicable up to $z=1.5$. Thus future surveys such as EUCLID and DESI will provide ideal data for the method presented in our current and previous works.

Previously we found that, for $1 / 8$-sky mock surveys that have 8 million galaxies and are sampled to have a roughly uniform number density in $z=0-1.5$, the AP effect results in tight constraints with $68.3 \%$ CL intervals of $\delta \Omega_{m} \sim 0.03$ and $\delta w \sim 0.1$ when using the AP test alone (not combining it with others). The constraints from DESI, which will probe 30 million galaxies and reach $z=1.5$, will be tighter than that.

It would be interesting to see whether we can detect firm evidence for a deviation of $w(z)$ from -1 in future surveys. This also demands us to make more precise correction of systematics, which would becomes comparable to or even larger than the statistical error.

The cosmological dependence of the systematics is negligible at the level of precision of current surveys, as can be seen in Appendix B. In future experiments at the next stage, the impact of a cosmological dependence of systematics would definitely be larger. If we assume the statistical error proportional to $1 / \sqrt{N}$ where $N$ is the number of galaxies, then future surveys, such as DESI, will have $\sim 6$ times smaller of a statistical error than SDSS-III. However, the cosmological dependence of the systematics could be easily solved by, e.g., interpolating among systematics estimated from several sets of simulations with different cosmologies, or considering theoretical estimation of systematics (H. Park et al. 2018, in preparation). We believe this would not be a big problem limiting the application of the method.

We expect the method will play an important role in deriving cosmological constraints from future spectroscopic galaxy surveys.

We thank Korea Institute for Advanced Study for providing computing resources (KIAS Center for Advanced Computation Linux Cluster System). C.G.S. acknowledges support from the National Research Foundation (NRF-2017R1D1A1B03034900). A.S. would like to acknowledge the support of the National Research Foundation of Korea (NRF-2016R1C1B2016478).

We thank Stephen Appleby, Seokcheon Lee, Maurice van Putten, Graziano Rossi, and Yi Wang for helpful discussions.

Based on observations obtained with Planck (http://www. esa.int/Planck), an ESA science mission with instruments and contributions directly funded by ESA Member States, NASA, and Canada.

Funding for SDSS-III has been provided by the Alfred P. Sloan Foundation, the Participating Institutions, the National Science Foundation, and the U.S. Department of Energy Office of Science. The SDSS-III web site is http://www.sdss3.org/. SDSS-III is managed by the Astrophysical Research Consortium for the Participating Institutions of the SDSS-III Collaboration including the University of Arizona, the Brazilian Participation Group, Brookhaven National Laboratory, Carnegie Mellon University, University of Florida, the French Participation Group, the German Participation Group, Harvard University, the Instituto de Astrofisica de Canarias, the Michigan State/Notre Dame/JINA Participation Group, Johns Hopkins University, Lawrence Berkeley National Laboratory, Max Planck Institute for Astrophysics, Max Planck Institute for Extraterrestrial Physics, New Mexico State University, New York University, Ohio State University, Pennsylvania State University, University of Portsmouth, Princeton University, the Spanish Participation Group, University of Tokyo, University of Utah, Vanderbilt University, University of Virginia, University of Washington, and Yale University.

\section{Appendix A \\ Approximating the 2PCFs in Cosmologies Other than the Fiducial One}

The number of galaxy pairs is counted in bins of separation $s$ and cosine of the angle with respect to the $\operatorname{LoS} \mu$, where the 


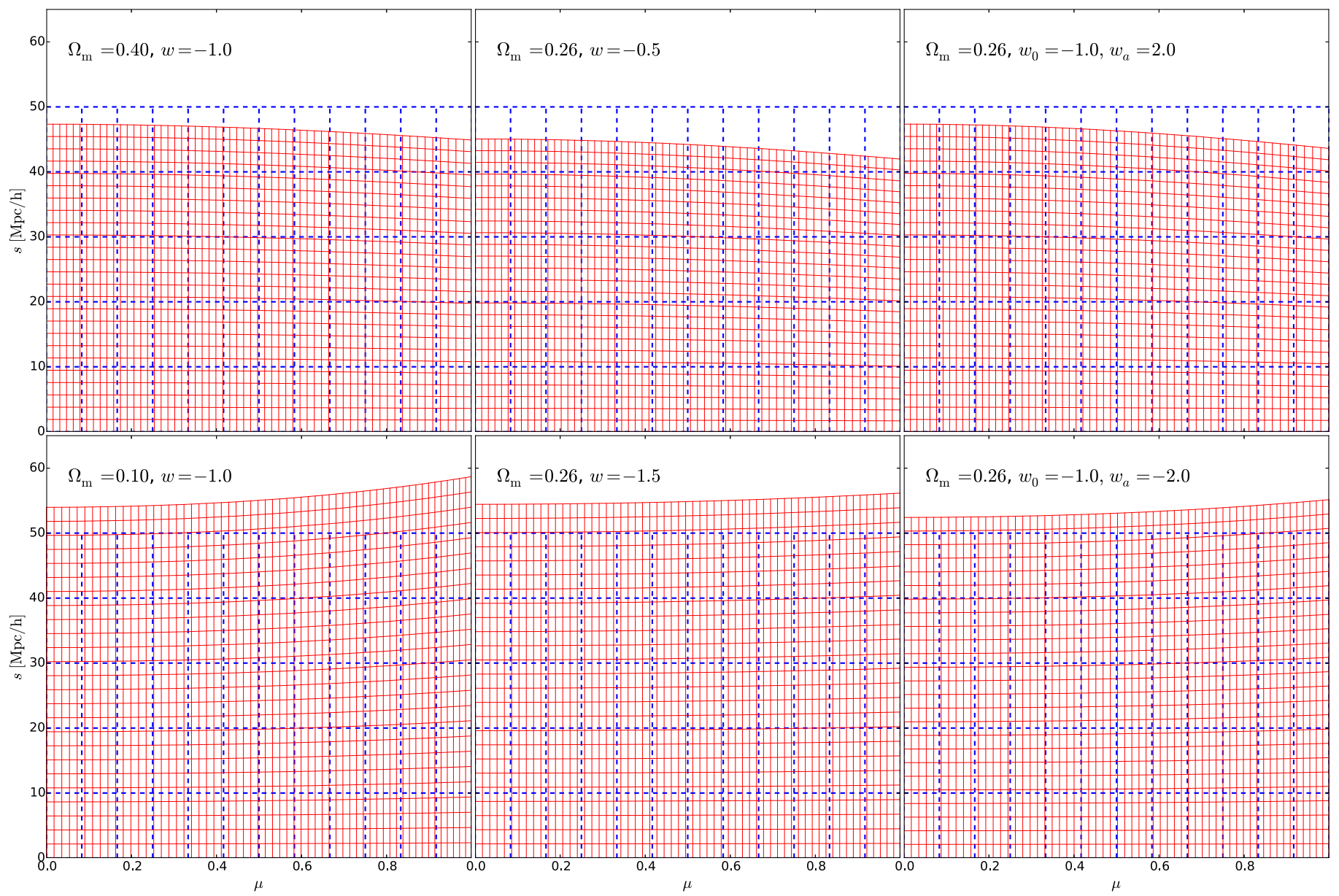

Figure 6. Mapping $\xi(s, \mu)$ from the fiducial cosmology (taken as the $\Omega_{m}=0.26 \Lambda \mathrm{CDM}$ cosmology) to six different cosmologies: $\left(\Omega_{m}, w_{0}, w_{a}\right)=(0.4,-1,0),(0.26$, $-0.5,0),(0.26,-1,2),(0.1,-1,0),(0.26,-1.5,0)$, and $(0.26,-1,-2)$. The number counts are measured in the fiducial cosmology in the blue dashed grid; in other cosmologies their distribution becomes the red solid grid (according to Equation (12)). We use this relation to obtain $\xi(s, \mu)$ in these non-fiducial cosmologies without remeasuring the number counts. To enhance the accuracy, we count the number of galaxy pairs in pixels that are five times smaller (the small red pixels), and group them together to infer the values of $\xi(s, \mu)$ in the blue dashed pixels (for illustration purposes, the blue and red grids are 10 times sparser than the grids adopted in the real analysis).

comoving positions were computed in a fiducial cosmology $\left(\Omega_{m}=0.26 \Lambda \mathrm{CDM}\right)$. These binned measurements are then translated from the "fiducial" cosmology to the measurements in a "target" cosmology using the following coordinate transforms:

$$
\begin{aligned}
& s_{\text {target }}=s_{\text {fiducial }} \sqrt{\alpha_{\|}^{2} \mu_{\text {fiducial }}^{2}+\alpha_{\perp}^{2}\left(1-\mu_{\text {fiducial }}^{2}\right)}, \\
& \mu_{\text {target }}=\mu_{\text {fiducial }} \frac{\alpha_{\|}}{\sqrt{\alpha_{\|}^{2} \mu_{\text {fiducial }}^{2}+\alpha_{\perp}^{2}\left(1-\mu_{\text {fiducial }}^{2}\right)}}
\end{aligned}
$$

where $\alpha_{\perp} \equiv D_{A \text {,target }} / D_{A, \text { fiducial }}, \alpha_{\|} \equiv H_{\text {fiducial }} / H_{\text {target }}$, and $D_{A}$ and $H$ are computed in the effective redshifts of the six redshift bins. In the fiducial cosmology we measure $\xi(s, \mu)$ with a high resolution of $\Delta s=0.2 \mathrm{Mpc} h^{-1}, \Delta \mu=1 / 600$, and later these small "pixels" are grouped to infer the number counts in other cosmologies, in large pixels of $\Delta s=1 \mathrm{Mpc} h^{-1}$ and $\Delta \mu=1 / 120$. In the case when one small pixel belongs to more than one larger pixel, a correction is applied by computing the fraction of the overlapping area. A dense grid of $\Delta s=0.2 \mathrm{Mpc}^{-1}, \Delta \mu=1 / 600$ can significantly reduce the edge effect; if we use Equation (12) to do a simple interpolation on a grid with $\Delta s=1 \mathrm{Mpc} h^{-1}$, $\Delta \mu=1 / 120$, the edge effect becomes so large that the derived cosmological constraints suffer from a significant error.
We compared the cosmological constraints derived from this approximation method with our results in Li et al. (2016), where we made the measurements in each cosmological model without approximation. Without considering the edge effect, they deviate from the original contour by more than $1 \sigma$. Whatever the fiducial cosmology, the error always exists since we always need to compute $\chi^{2}$ of non-fiducial cosmological parameters when making the contour. The amplitude of error is found to be larger if the fiducial cosmology is far from the constrained region of parameter space. For example, in the case that the deviation is as large as $\delta \Omega_{m}>0.2$ and $\delta w>2$, the change in the position and size of the contour is $\sim 10 \%$.

The above procedure is illustrated in Figure 6. Using the relations given by Equation (12), we obtained the distribution of number counts in cosmologies other than the fiducial cosmology. We ensure the accuracy of the remapping by performing the pair counting using pixels that are five times smaller (the small red pixels), and regrouping these together to infer the number counts at the desired resolution (the large blue dashed pixels).

Figure 3 plots the approximate 2PCFs in the cosmology with $w_{a}=-2$ and evolving dark energy. We find that the approximation procedure only introduces a $\lesssim 0.5 \%$ error in $\hat{\xi}_{\Delta s}(\mu)$, which is 10 times smaller than the intrinsic noise (the Poisson noise and cosmic variance) in $\hat{\xi}_{\Delta s}(\mu)$. So it should be 

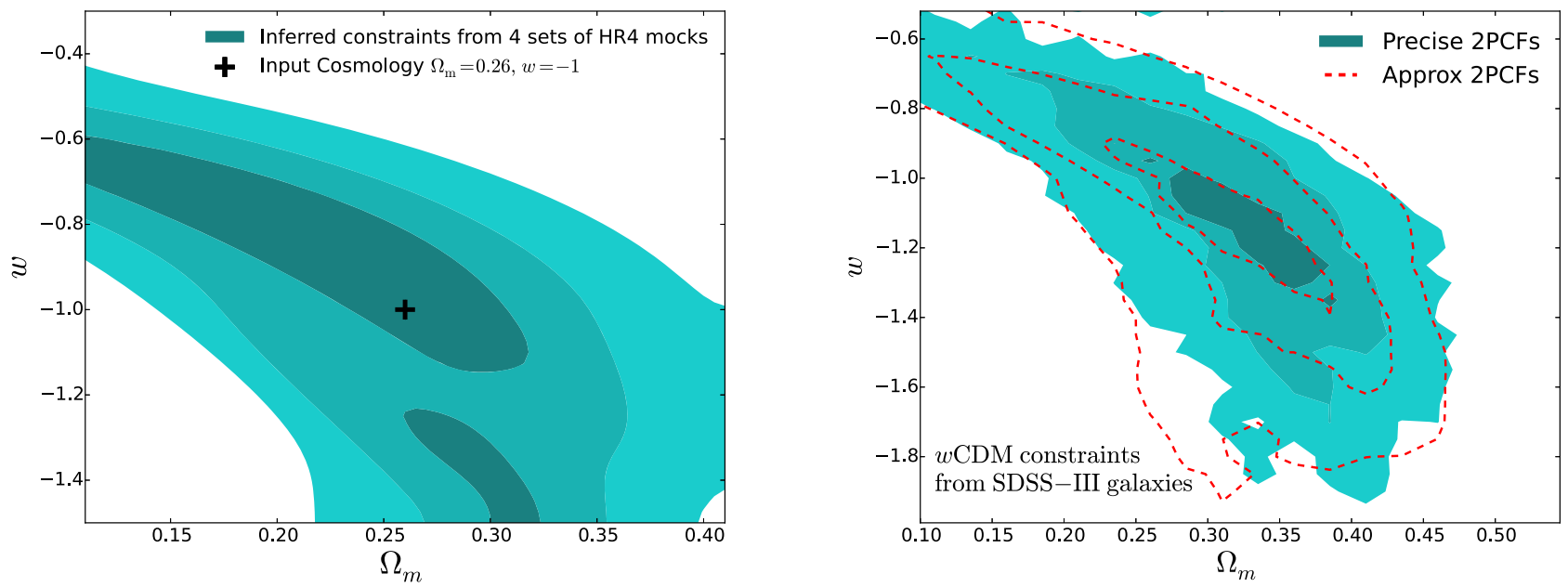

Figure 7. Left panel: input-output test of the AP methodology using four sets of BOSS DR12 galaxy mock catalogs constructed from the HR4 simulation. The approximate $2 \mathrm{PCF}$ are adopted in the analysis. The inferred cosmological constraints from the method, shown by the cyan contours, are consistent with the input cosmology (the simulation cosmology, i.e., the $\Omega_{m}=0.26 \Lambda \mathrm{CDM}$, marked by the black plus sign). Right panel: cosmological constraints from the BOSS DR12 galaxies, assuming a $w \mathrm{CDM}$ cosmology (i.e., the value of $w_{a}$ fixed as zero). Results obtained using the precise 2PCFs (cyan filled) and approximate 2PCFs (red dashed) agree with each other quite well.

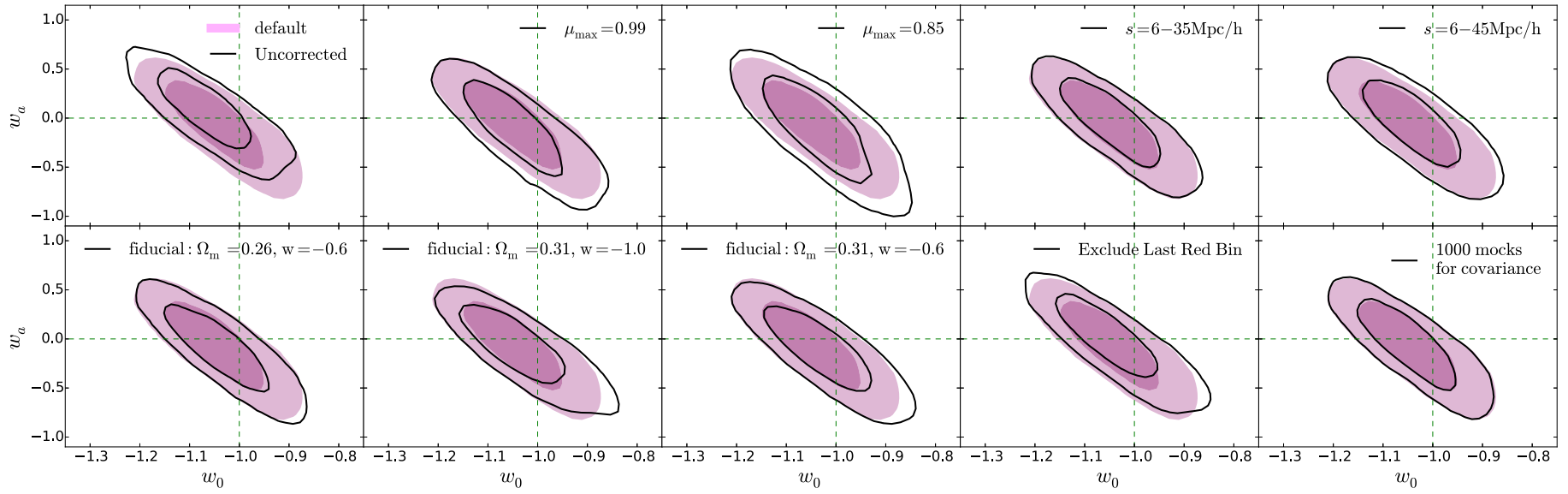

Figure 8. Robustness test of the results. The "default" constraints (magenta contours) are derived using six redshift bins, $\mu_{\max }=0.97, s=6-40 \mathrm{Mpc} h^{-1}$, a fiducial cosmology $\Omega_{m}=0.26, w=-1.0$ for the approximation of 2PCF, systematic effects estimated from Horizon Run 4 simulations, and covariance estimated using 2000 MultiDark PATCHY mocks. When we alter one of these options by discarding the systematic correction, using $\mu_{\max }=0.99, \mu_{\max }=0.85, s=6-35 \mathrm{Mpc} h^{-1}$, $s=6-45 \mathrm{Mpc}^{-1}$, a fiducial cosmology of $\Omega_{m}=0.26, w=-0.6$, excluding the last redshift bin in the analysis, or reducing the number of mocks in the estimation of covariance matrix, the results remain robust (black contours).

precise enough to use the approximate $2 \mathrm{PCF}$ in the statistical analysis.

We performed a series of tests to check the reliability of using the approximate $2 \mathrm{PCF}$ in the cosmological analysis. An inputoutput test was conducted using the four sets of mock catalogs of BOSS DR12 galaxies constructed from HR4 (Kim et al. 2015). The results are shown in the left panel of Figure 7. The input cosmology is the simulation cosmology, i.e., $\Omega_{m}=0.26 \Lambda \mathrm{CDM}$. It lies within the $1 \sigma$ contour of the inferred constraints. The right panel of Figure 7 displays the cosmological constraints from real observational data when fixing $w_{a}$ as zero (hereafter $w \mathrm{CDM}$ ). Results obtained using the precise and approximate $2 \mathrm{PCF}$ agree quite well with each other.

\section{Appendix B Robustness Check}

Figures 8 and 9 show that the derived constraints are almost unaffected if we discard the systematics correction. This

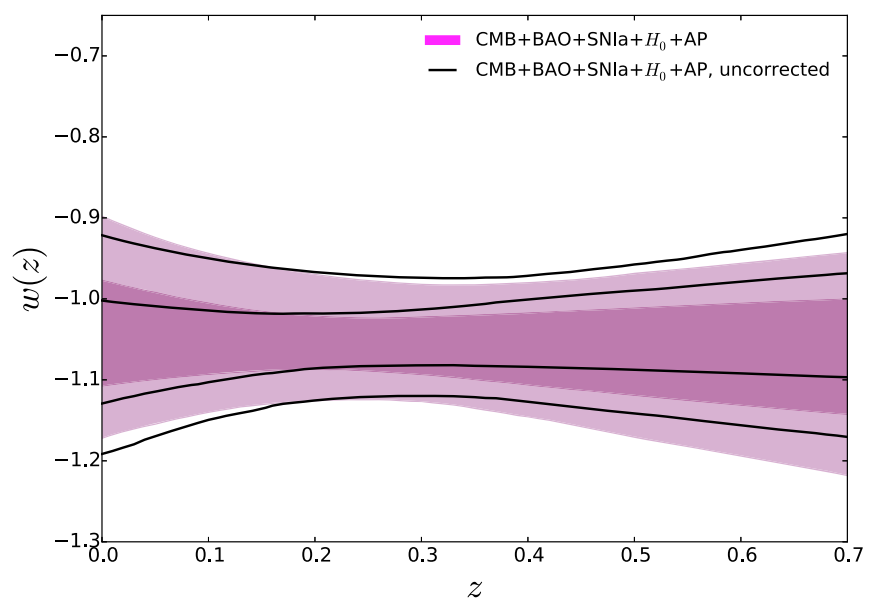

Figure 9. Derived redshift evolution of $w(z)$ from $\mathrm{CMB}+\mathrm{BAO}+\mathrm{SNe} \mathrm{Ia}+$ $H_{0}+$ AP. There is no significant change if we discard the systematics correction procedure in the analysis using the AP method. 
indicates that, for the data analysis of current galaxy surveys, the systematic effects in our method are not significant. But it remains to be seen whether this is true for future galaxy surveys, or when the cosmology dependence of the systematics effects is taken into account.

Furthermore, Figure 8 shows that the result is unaffected by the $\operatorname{LoS} \mu$-cut, the range of radial integration, the choice of fiducial cosmology in the mapping of $\xi(s, \mu)$, or the number of mocks. The result does not change significantly if we remove the highest redshift bin, where the estimated systematics are comparably large. This further justifies our conclusion that the effect of systematics is not significant in this analysis.

\section{ORCID iDs}

Xiao-Dong Li (ib https://orcid.org/0000-0003-3964-0438 Cristiano G. Sabiu (ib https://orcid.org/0000-0002-5513-5303 Changbom Park (10 https://orcid.org/0000-0001-9521-6397 Gong-bo Zhao (i) https://orcid.org/0000-0003-4726-6714 Hyunbae Park (iD https://orcid.org/0000-0002-7464-7857 Arman Shafieloo (i) https://orcid.org/0000-0001-6815-0337 Juhan Kim (10) https://orcid.org/0000-0002-4391-2275 Sungwook E. Hong (i) https://orcid.org/0000-0003-4923-8485

\section{References}

Ade, P. A. R., Aghanim, N., Arnaud, M., et al. 2016, A\&A, 594, 13 Alam, S., Ata, M., Bailey, S., et al. 2017, MNRAS, 470, 2617 Albrecht, A., Bernstein, G., Cahn, R., et al. 2006, arXiv:astro-ph/0609591 Alcock, C., \& Paczynski, B. 1979, Natur, 281, 358

Anderson, L., Aubourg, É., Bailey, S., et al. 2014, MNRAS, 441, 24 Ballinger, W. E., Peacock, J. A., \& Heavens, A. F. 1996, MNRAS, 282, 877 Betoule, M., Kessler, R., Guy, J., et al. 2014, A\&A, 568, 22
Beutler, F., Blake, C., Colless, M., et al. 2011, MNRAS, 416, 3017

Blake, C., Glazebrook, K., \& Davis, T. M. 2011, MNRAS, 418, 1725

Bueno Belloso, A., Pettinari, G. W., Meures, N., \& Percival, W. J. 2012, PhRvD, 86, 023530

Chevallier, M., \& Polarski, D. 2001, IJMPD, 10, 213

Efstathiou, G. 2014, MNRAS, 440, 1138

Hamaus, N., Pisani, A., Sutter, P. M., et al. 2016, PhRvL, 117, 091302

Hartlap, J., Simon, P., \& Schneider, P. 2007, A\&A, 464, 399

Hong, S. E., Park, C., \& Kim, J. 2016, ApJ, 823, 103

Jackson, J. 1972, MNRAS, 156, 1

Jennings, E., Baugh, C. M., \& Pascoli, S. 2011, MNRAS, 420, 1079

Joyce, A., Jain, B., Khoury, J., \& Trodden, M. 2015, PhR, 568, 1

Kaiser, N. 1987, MNRAS, 227, 1

Kim, J., Park, C., L'Huillier, B., \& Hong, S. E. 2015, JKAS, 48, 213

Kitaura, F. S., Rodríguez-Torres, S., Chuang, C.-H., et al. 2016, MNRAS, 456, 4156

Klypin, A., Yepes, G., Gottlober, S., Prada, F., \& Hess, S. 2016, MNRAS, 457,4340

Komatsu, E., Smith, K. M., Dunkley, J., et al. 2011, ApJS, 192, 18

Lavaux, G., \& Wandelt, B. D. 2012, ApJ, 754, 109

Lewis, A., \& Bridle, S. 2002, PhRvD, 66, 103511

Li, M., Li, X.-D., Wang, S., \& Wang, Y. 2011, CoTPh, 56, 525

Li, X.-D., Park, C., Forero-Romero, J., \& Kim, J. 2014, ApJ, 796, 137

Li, X.-D., Park, C., Sabiu, C. G., et al. 2016, ApJ, 832, 103

Li, X.-D., Park, C., Sabiu, C. G., \& Kim, J. 2015, MNRAS, 450, 807

Linder, E. V. 2003, PhRvL, 90, 091301

Mao, Q., Berlind, A. A., Scherrer, R. J., et al. 2017, ApJ, 835, 160

Marinoni, C., \& Buzzi, A. 2010, Natur, 468, 539

Outram, P. J., Shanks, T., Boyle, B. J., et al. 2004, MNRAS, 348, 745

Park, C., \& Kim, Y.-R. 2010, ApJL, 715, L185

Percival, W. J., Ross, A. J., Sánchez, A. G., et al. 2014, MNRAS, 439, 2531

Reid, B., Ho, S., Padmanabhan, N., et al. 2016, MNRAS, 455, 1553

Riess, A. G., Macri, L., Casertano, S., et al. 2011, ApJ, 730, 119

Ross, A. J., Samushia, L., Howlett, C., et al. 2015, MNRAS, 449, 835

Ryden, B. S. 1995, ApJ, 452, 25

Yoo, J., \& Watanabe, Y. 2012, IJMPD, 21, 1230002

Zhang, X., Huang, Q.-G., \& Li, X.-D. 2018, arXiv:1801.07403 